Cochrane Database of Systematic Reviews

\title{
Interventions for psychosexual dysfunction in women treated for gynaecological malignancy (Review)
}

Flynn P, Kew F, Kisely SR

Flynn P, Kew F, Kisely SR.

Interventions for psychosexual dysfunction in women treated for gynaecological malignancy.

Cochrane Database of Systematic Reviews 2009, Issue 2. Art. No.: CD004708.

DOI: 10.1002/14651858.CD004708.pub2.

www.cochranelibrary.com 
TABLE OF CONTENTS

ABSTRACT

PLAIN LANGUAGE SUMMARY

BACKGROUN

OBJECTIVES

METHODS

RESULTS

DISCUSSION

AUTHORS' CONCLUSIONS

ACKNOWLEDGEMENTS

REFERENCES

CHARACTERISTICS OF STUDIES

DATA AND ANALYSES

Analysis 1.1. Comparison 1 Vaginal oestrogen versus placebo, Outcome 1 Dyspareunia in all patients.

Analysis 1.2. Comparison 1 Vaginal oestrogen versus placebo, Outcome 2 Dyspareunia in sexually active.

Analysis 2.1. Comparison 2 Brachytherapy $0.4 \mathrm{~Gy} / \mathrm{Hr}$ versus $0.8 \mathrm{~Gy} / \mathrm{hr}$, Outcome 1 Dyspareunia in all patients.

Analysis 2.2. Comparison 2 Brachytherapy $0.4 \mathrm{~Gy} / \mathrm{Hr}$ versus $0.8 \mathrm{~Gy} / \mathrm{hr}$, Outcome 2 Dyspareunia at 25 months post treatment. ...

Analysis 3.1. Comparison 3 Clinical Nurse Specialist versus Standard Care, Outcome 1 Not sexually active.

Analysis 3.2. Comparison 3 Clinical Nurse Specialist versus Standard Care, Outcome 2 Previously active, unsatisfactory now. ... APPENDICES

WHAT'S NEW

HISTORY

CONTRIBUTIONS OF AUTHORS

DECLARATIONS OF INTEREST

DIFFERENCES BETWEEN PROTOCOL AND REVIEW

INDEX TERMS 
[Intervention Review]

\section{Interventions for psychosexual dysfunction in women treated for gynaecological malignancy}

Paul Flynn¹, Fiona Kew², Steve R Kisely ${ }^{3}$

1Obstetrics and Gynaecology, Singleton Hospital, Swansea, UK. ${ }^{2}$ Gynaecological Oncology, Sheffield Teaching Hospitals, Sheffield, UK. ${ }^{3}$ School of Medicine, The University of Queensland, Woolloongabba, Australia

Contact: Paul Flynn, Obstetrics and Gynaecology, Singleton Hospital, Swansea, UK. paulmflynn67@me.com, paul@obsngobs.com.

Editorial group: Cochrane Gynaecological, Neuro-oncology and Orphan Cancer Group.

Publication status and date: Edited (no change to conclusions), published in Issue 9, 2019.

Citation: Flynn P, Kew F, Kisely SR. Interventions for psychosexual dysfunction in women treated for gynaecological malignancy. Cochrane Database of Systematic Reviews 2009, Issue 2. Art. No.: CD004708. DOI: 10.1002/14651858.CD004708.pub2.

Copyright $@ 2019$ The Cochrane Collaboration. Published by John Wiley \& Sons, Ltd.

\section{A B S T R A C T}

\section{Background}

Psychosexual dysfunction (sexual difficulties not directly due to physical factors) is known to be a common complication of treatment for gynaecological cancer. It has a considerable impact on quality of life (QoL) for the increasing number of women who are survivors of gynaecological cancer.

\section{Objectives}

To determine the effectiveness of interventions for psychosexual dysfunction in women who have been treated for gynaecological malignancy (cancer of uterine cervix, uterine corpus, ovary, vulva).

\section{Search methods}

We searched the Cochrane Central Register of Controlled Trials (CENTRAL, up to October 2008), MEDLINE (1950 to October 2008), EMBASE (1982 to October 2008), CINAHL (1980 to October 2008) and PsycINFO (1806 to October 2008). We hand searched reference lists from eligible trials.

\section{Selection criteria}

We selected all randomized controlled trials (RCTs) of a medical or psychological intervention to prevent or treat psychosexual dysfunction in adult women previously treated for gynaecological cancer.

\section{Data collection and analysis}

We selected five studies for inclusion in this review and analysed any outcome data relating to resumption of sexual intercourse, DSM-IV diagnoses or validated scales of sexual functioning. Sensitivity analysis was performed where possible.

\section{Main results}

The review included data from 5 studies, comprising a total of 413 patients, examining 5 different interventions. One trial suggested a short-term benefit for the use of vaginal Dienoestrol in women after pelvic radiotherapy (NNT = 4). Another trial suggested a short-term benefit for one regime of low dose-rate brachytherapy over another but this modality is not in widespread use. Studies of a Clinical Nurse Specialist intervention, Psychoeducational Group Therapy and a Couple-Coping intervention, did not show any significant benefit. All the studies were of poor methodological quality. 


\section{Authors' conclusions}

There is no convincing evidence to support the use of any interventions for psychosexual dysfunction in women treated for gynaecological cancer. There is a need for more studies of high methodological quality.

\section{PLAIN LANGUAGE SUMMARY}

\section{Interventions for psychosexual dysfunction in women treated for gynaecological malignancy}

This systematic review found very few good quality trials of interventions to treat or prevent psychosexual problems occurring in women after treatment for gynaecological cancer. They were mostly small studies and all examined different types of interventions. This review found only weak evidence to support the use of vaginal oestrogen, some low dose-rate brachytherapy regimes and a number of psychological interventions. 


\section{B A C K G R O U N D}

Gynaecological cancers (cancer of the vulva, cervix, uterus, and ovary) can have a profound effect on the sexual function of women (Corney 1992; Corney 1993; Crowther 1994). The treatment for many is radical surgery, which can be both physically and psychologically mutilating. For example, women with cervical cancer who have a radical hysterectomy or pelvic radiotherapy may experience shortening of the vagina and absence of lubrication during intercourse resulting in pain (Bergmark 1999a). Although the mutilation caused by radical vulvar surgery is profound, women may also suffer adverse effects on sexual functioning from the treatment of cancer of the ovary or uterus by hysterectomy, oophorectomy and chemotherapy and the resulting oestrogen deficiency. Impaired sexual function is undoubtedly multifactorial and psychological reactions to gynaecological cancer can cause problems with sexual functioning. These may include: disturbances of mood, gender and sexual identity, and body-image; loss of control over body systems; disturbance of normal opportunities for intimate behaviour; and loss of fertility (Weijmar-Schultz 1990). Even the development of symptoms of gynaecological cancer and its diagnosis have been associated with adverse effects on sexual function (Anderson 1986).

Up to $63 \%$ of patients over 55 years and $37 \%$ of those under 55 years of age treated for gynaecological cancer are not sexually active after treatment (Thranov 1994). Even allowing for the obvious confounders of age and prior sexual activity, this measure may be an underestimate of sexual problems after gynaecological cancer. Studies based on post-treatment report of symptoms have found rates of sexual problems ranging from six to $100 \%$ (Weijmar-Schultz 1990). Since sexuality itself is not an objective measure, it follows that the assessment of sexual function is subjective and must be operationalized based on ideal and real experiences. Restarting sexual activity is not synonymous with resumption of a fulfilling sexual relationship, just as the anatomical possibility of sexual function does not guarantee resumption of satisfactory sexual activity. Much research does assume an existing heterosexual relationship, raising doubts about its appropriateness for women in lesbian relationships or those not in a relationship at all.

Since the concept of sexuality for women is broader than anatomical function and encompasses effects on relationships and sexual self-concept (Butler 1998), psychosexual therapies have been utilised to help restore satisfactory sexual function. To our knowledge there have been no other systematic reviews of this topic and so it is appropriate for the effectiveness of those therapies which have thus far been subjected to controlled trials to be assessed.

\section{O B JE C T IVES}

To determine the effectiveness of interventions for psychosexual dysfunction in women who have been treated for gynaecological malignancy.

\section{METHODS}

\section{Criteria for considering studies for this review}

\section{Types of studies}

Randomised controlled trials (RCTs) of any form of physical, psychological and/or pharmacological treatment were eligible for consideration providing that they compared the intervention either with another treatment, placebo treatment or non-intervention (e.g. waiting list controls).

\section{Types of participants}

Participants in the studies had to be exclusively female or the data for female subjects had to be accessible. All had to have had a primary malignancy of the female genital tract i.e. vulva, vagina, cervix, corpus uteri, fallopian tube or ovary. Those with metastases in the genital tract from an extra-genital primary were excluded as they differ considerably in treatment and prognosis from primary gynaecological malignancies.

Participants were eligible for inclusion if they were aged over 16 years and had demonstrable psychosexual dysfunction or distress at entry to the study. These could include the DSMIV diagnoses of Dyspareunia (302.76), Female Orgasmic Disorder (302.73), Female Sexual Arousal Disorder (302.72), Hypoactive Sexual Desire Disorder (302.71), Sexual Aversion Disorder (302.79) and Vaginismus (306.51) (APA 1994).

The method of diagnosis could include the sexual activity questionnaire (Stead 1999) as well as instruments of Quality of Life (QoL) and psychological symptoms such as the Short Form 36 Hamilton Rating Scale (Ware 1993), Hospital Anxiety and Depression Scale (Zigmond 1983), General Health Questionnaire (Goldberg 1997) and the Beck Depression Inventory (Steer 1999).

\section{Types of interventions}

Any psychological or medical interventions to prevent or treat psychosexual dysfunction were considered in this review. Psychological treatments such as educational therapies, behavioural techniques and cognitive therapies were considered along with hybrid therapies of one or more of these combined with another intervention. Medical treatments considered included vaginal oestrogen therapy, different radiotherapy regimes to try to ameliorate their effects on sexual function, the use of vaginal dilators to maintain vaginal capacity and the use of a clitoral therapy device to improve vaginal blood flow.

\section{Types of outcome measures}

To be included for consideration studies had to have as an outcome measure the resumption of sexual activity as measured by selfreport, partner report, or sexual activity questionnaire. Since the resumption of sexual activity alone does not necessarily equate to a satisfying relationship, improvement on any form of rating scale validated in a comparable patient set was also accepted as a valid outcome measure. 


\section{Search methods for identification of studies}

\section{Electronic searches}

1. Initially the Cochrane Review Group's Specialised register was checked to identify all potentially eligible studies (Last checked 18/10/08).

2. The following electronic databases were searched to identify potentially eligible studies and review articles: CENTRAL (Cochrane Library, MEDLINE (1950 to October 2008), CINAHL (1982 to October 2008), EMBASE (1980 to October 2008), PsycINFO (1806 to October 2008), the Database of Abstracts of Reviews of Effectiveness (DARE), and Biological Abstracts (January 1980 to October 2008). Searches were carried out using the online search engine OvidSP and utilised the filter for RCTs as described in the Cochrane Handbook for Reviewers (Cochrane Handbook). Any potentially relevant non-English language papers were translated.

3. The search strategy for OvidSP is in Appendix 1.

\section{Searching other resources}

1. The reference lists of all potentially relevant articles retrieved, as well as those of systematic reviews, were checked to identify other potentially relevant articles. These articles were retrieved and assessed for possible inclusion in the review.

2. Personal communications - the lead author of all relevant papers identified was emailed or written to in order to ascertain if they knew of any additional published or unpublished studies that might be relevant to the review.

The search strategies have been developed and executed by the author.

\section{Data collection and analysis}

\section{Selection of studies}

Trials were selected for inclusion in the review after independent assessment by two review authors and additional information was sought, if required, from the authors of the selected trials.

Two review authors (FK and PF) independently selected suitable studies for inclusion in this review as detailed below. Where the two review authors disagreed about the inclusion of a study, disagreements were resolved by a consensus of opinion, and although a third review author (SK) was available for arbitration, this was not required.

The titles and abstracts of studies identified by searching electronic databases were assessed to determine whether each article met the eligibility criteria. In order to prevent any bias, a list of all titles and abstracts was printed out excluding the authors' names, institutions and journal titles. If a title and abstract contained sufficient information to determine that the article did not meet the inclusion criteria, then it was rejected. A record of all rejected papers was kept and the reasons for rejection were documented.

The full papers of all remaining titles and abstracts deemed relevant were then retrieved. In addition, all other potentially relevant articles identified by the various search strategies (reference checking, personal communications etc) were also reviewed. Any potentially relevant papers in languages other than English were translated and reviewed by someone who spoke the language. All articles were reviewed independently by two of the review authors, who completed a form for each study and scored the quality of the research as defined below. The reasons for exclusion were documented. Where the same study had more than one article written about the outcomes, all articles were treated as one study and the results were presented only once.

\section{Data extraction and management}

The two review authors (PF, FK) completed the extraction of data from the papers on to a form to elicit the following information:

- general: published/unpublished, title, authors, source, contact address, country, language of publication, year of publication, duplicate publications, sponsoring, setting (hospital inpatients, primary care, community)

- trial characteristics: design, duration, randomisation and method, allocation concealment and method, blinding of outcome assessors, check of blinding

- interventions: frequency, timing, comparison interventions, comedications

- patient characteristics: sampling, exclusion criteria, number of participants, age, sex, ethnicity, marital status, educational status, duration of symptoms, number of complications, similarity of groups at baseline (including any co-morbidity), withdrawals/losses to follow-up (reasons/descriptions)

- type of psychiatric co-morbidity: clinical diagnosis or symptomatology assessed by questionnaires

- type of assessment tool used to assess quality of life psychiatric co-morbidity: e.g. Beck Depression Inventory, Zung Depression scale, Hospital Anxiety and Depression Scale, Structured Interview, DSM-IV criteria

- type of intervention: psychological, pharmacological, medical or surgical; usual care versus intervention; 'attention' placebo versus intervention.

- type of outcomes: resumption of sexual activity, as measured by self report interview or sexual activity questionnaire and possibly verified by the partner, improvement in relevant validated scales of QoL and psychological symptoms

- timing of follow-up: short, medium or long-term

- assessment of methodological quality: method of randomisation used, if stated, method of allocation concealment (adequate, unclear, inadequate or allocation concealment not used); blinding of outcome assessors (yes, no, unclear); and patients lost to follow-up (cut off 20\% attrition or more); intention to treat analysis.

\section{Assessment of risk of bias in included studies}

Assessment of the quality of a particular trial will be made in accordance with guidelines in the Cochrane Handbook.

\section{Assessment of the method of randomisation}

To prevent selection bias, someone who is not responsible for recruiting the participants, such as a central trial office or someone not involved in the trial, should conduct the randomisation. The method of randomisation was noted on the data extraction form. 


\section{Assessment of the degree of blinding (treatment and outcome} assessment) and allocation concealment

Allocation concealment was assessed as described in the Cochrane Handbook. This is as follows:

A - adequate description of the allocation procedure,

B - unclear description of the allocation procedure,

C - inadequate description of the allocation procedure and

D - allocation concealment was not used.

If the review authors disagreed over which category a trial was allocated to, resolution was attempted by discussion or by obtaining further information. In addition, review authors were blinded to the authors' names, institutions and journal title to prevent any bias.

\section{Losses to follow-up}

The paper should have given an adequate description of the loss of its participants in terms of the number of withdrawals, dropouts and protocol deviations. Had any study had losses to follow-up of more than $20 \%$ of those originally randomised, these data would have been presented as a sub-group, but this was not required.

\section{Assessment of reporting biases}

Had the trial interventions been directly comparable the data would have been entered into a funnel plot (size of study versus size of effect) (Egger 1997) to attempt to detect the possibility of publication bias. Since the identified studies all addressed different interventions this was not assessable.

\section{Data synthesis}

\section{Data Entry}

Data was entered into RevMan software by PF and duplicated by FK. The data extracted from included studies was summarised.

\section{Data Types}

Depending on the type of study the outcomes were assessed using continuous (for example, changes in depression scales), categorical (for example, one of three categories on a QOL scale, such as 'better', 'worse', or 'no change'), or dichotomous (for example either sexually active or not sexually active) measures.

Continuous data: Many rating scales are available to measure outcomes in psychological trials. These scales varied in the quality of their validation and reliability. Therefore, if a rating scale's validation had not been published in a peer-reviewed journal then the data was not included in this review. In addition, the rating scale should have been either self-report or completed by an independent observer or relative. It was planned that any trials that had used the same instrument to measure specific outcomes were compared directly. If continuous data was presented from different scales rating the same effect both sets of data were to be presented and the general direction of the effect inspected. The mean and standard deviation (SD) were reported. Where SDs were not reported in the paper, attempts were made to obtain them from the authors or to calculate them using other measures of variation that are reported, such as the confidence intervals (Cls). If possible, meta-analysis was considered for data from different scales rating the same effect using the Standardised Mean Difference (SMD).
Dichotomous data: Continuous outcome measures were converted to dichotomous data where necessary. If the authors of the study used a designated cut-off point for determining clinical effectiveness the review authors used this where appropriate. Otherwise, cut-offs on rating scales were identified and participants were divided on the basis of whether they were 'clinically improved' or 'not clinically improved'. For dichotomous outcomes, a MantelHaenszel odds ratio (OR) with its associated $95 \% \mathrm{Cl}$ was estimated. As a summary measure of effectiveness, where possible the number needed to treat statistic (NNT) was also calculated.

\section{Subgroup analysis and investigation of heterogeneity}

In the review protocol sub group analyses were planned to consider:

- Differences between studies, which define sexual activity, QOL, and psychiatric symptoms operationally (clinician diagnosis or validated questionnaire and whether validated in this specific population or in other groups).

- Differences between studies that include partner's views and those that do not.

- Differences between types of interventions (psychological, pharmacological, medical or surgical) and types of controls.

- Differences between well-defined and less well-defined psychological interventions.

As the studies included in this review did not lend themselves to aggregation, sub-group analysis was not possible. A review of heterogeneity was not required since only one trial was identified for each type of intervention.

\section{Sensitivity analysis}

The reliability of the conclusions of the review was tested with sensitivity analyses of the data where possible. The data for each study was re-analysed using different models (fixed-effect instead of random-effect), altering substitutions for any missing data, and excluding trials of low methodological quality. If any of these had changed the results significantly then the conclusions might have been of lesser validity.

\section{RES U L T S}

\section{Description of studies}

\section{Results of the search}

A search of electronic databases using the previously described search strategy yielded 1559 citations. Examination of the titles and abstracts of these produced 12 that were potentially eligible but examination of the full text of these articles allowed us to exclude 7 non-RCT or uncontrolled studies. Examination of the reference lists of the five remaining eligible studies yielded one additional study that was published as an abstract only. Correspondence with the author who had recently retired did not yield sufficient information for it to be included in the review. Further information was requested from the authors of the five studies above but only one reply was received, which did not provide any more useful information. As some of these studies were published some time ago, the corresponding authors may have moved to another institution or retired. Should any further information be obtained from them it will be included in future review updates. 


\section{Included studies}

\section{Topical oestrogen prophylaxis for post-Irradiation vaginal complications}

A small $(n=93)$ placebo-controlled study in 1971 evaluated the vaginal administration of Dienoestrol $0.01 \%$ cream after completion of primary radiotherapy for carcinoma of the cervix (Pitkin 1971). Patient report of dyspareunia was just one of the outcomes examined, as well as vaginal bleeding, with other outcomes being assessed by clinical examination. Satisfaction with intercourse, or any other aspect of sexual function was not examined in this study that took a rather anatomical approach to sexuality. No baseline assessment of sexual function was performed but the reported rate of sexual activity (60\%) is rather low compared to other studies, although this may have been more typical of its era.

\section{Low dose-rate brachytherapy regimens in cervix cancer}

One French study examined the morbidity outcomes in a trial population $(n=204)$ where two different dose-rates $(0.4 \mathrm{~Gy} / \mathrm{hr}$ and $0.8 \mathrm{~Gy} / \mathrm{hr}$ ) for pre-operative vaginal brachytherapy were used prior to radical hysterectomy (Haie-Meder 1994). A previous publication reported the results with regard to mortality and recurrence (which were similar in both arms) so it is likely that the morbidity outcomes were not a primary outcome of the study. The method of treatment is one that is not in widespread use in the UK, the use of remote after-loading devices delivering high dose-rate therapy having superseded low dose rate brachytherapy. Similarly, the use of radiotherapy in a neoadjuvant setting such as this is not routine in UK practice but may be in other countries. Low dose-rate therapy is in use in certain countries including France and the conclusions of this study are of relevance for these areas.

\section{Clinical nurse specialist intervention}

In this small UK single-blind RCT of patients of mixed tumour sites, the intervention arm received a pre-surgery consultation with a psychosexually-trained gynaecological oncology specialist nurse and were visited by her at home on an average of three further occasions after surgery (Maughan 2001). The intervention is not described in detail and no checks on adherence to the intervention by the therapist were described. The control arm received standard nursing care only but were referred to specialist services if they reported any problems at follow-up visits. A qualitative study based on patient interviews was conducted in parallel with the RCT, but it should be noted that they interviewed nearly twice as many from the control group as the intervention group. Since the interviews could have had some therapeutic effect, this is a potential limitation of this study. The EORTC QLQ-30 questionnaire and the Lasry Sexual Functioning Scale were used to assess the outcomes.

\section{Psychoeducational group therapy}

A single small $(n=40)$ RCT trial conducted in Canada had as its primary outcome whether the intervention would improve compliance with the use of vaginal dilators after radiotherapy treatment for FIGO stage 1 or 2 cervix or corpus cancer (Robinson 1999). A variety of other treatments were received and, no doubt due to the small size of this study, the arms were not well balanced for other treatments. The mean age of the control group was also higher (51 versus 43 ) but the groups were similar in other respects. The intervention arm received two 90-minute group counselling sessions based on the Intervention-Motivation-Behavioural Skills model while the controls had a single one-to-one meeting with a counsellor who provided an information leaflet. There is no description of the level of training of the therapists and although there is some description of the information component, it is unclear whether there was a prescribed methodology for the other components and, if so, whether any checks on therapist adherence were performed. Follow-up was carried out by means of selfcompletion of the Sexual History Form by post and a global score was calculated (SHF-GS).

\section{Couple-Coping Intervention.}

This study from Australia randomised 94 women with breast and gynaecological cancers to one of three treatment arms medical information education; patient coping training; or CanCope, a couple coping intervention (Scott 2004). Those receiving medical information education received booklets and five 15-minute telephone calls in the 9 months after surgery. Patient coping training consisted of four 2-hour counselling sessions in the patient's home followed up by two 30-minute telephone calls. CanCope involved the patient and her partner in five 2-hour counselling sessions in their home followed by two 30-minute telephone calls. The therapists were extensively experienced in this field and followed a treatment manual. Sessions were audiotaped and a random selection reviewed to ensure adherence with protocols. The outcome was assessed using self-administered questionnaires returned by post, including the Brief Index of Sexual Function (BISF) and the Psychosocial Adjustment to Illness - Self Report (PAIS-SR) Sexual Difficulties subscale.

\section{Risk of bias in included studies}

The five studies included were all of poor methodological quality. The reasons for the decision in each case are explained below.

\section{Topical oestrogen prophylaxis for post-irradiation vaginal complications}

This study is unclear regarding the precise method of randomisation used, but the allocation concealment was graded as $A$, with the allocation only being revealed after completion of the trial. A placebo cream was used that was provided by the manufacturer and was identical in both presentation and composition (other than the active ingredient dienosetrol) to the treatment cream. Those assessing outcomes were also blinded, although the method of assessment (patient symptom report to doctor) is likely to have poorer ascertainment than methods such as validated questionnaires. The authors do not report any losses to follow-up and assume $100 \%$ compliance with treatment and as a result have performed an intention to treat (ITT) analysis without describing it as such. Such a compliance rate is surprising and is a weakness of this study. During sensitivity analysis, the inclusion of all trial participants in analysis led to the treatment effect becoming non-significant creating some doubt on the reliability of the findings. Overall this trial is not of high methodological quality.

\section{Low dose-rate brachytherapy in cervical cancer}

Much of the methodology of this trial is unclear and further clarification is awaited. The method of randomisation is unclear, although the allocation concealment is described and appears to have been adequate (grade A). Assessors were blinded to the outcomes but the method of ascertainment of complications was 
not described in detail and may have tended to underestimate adverse events. No mention is made of losses to follow-up or protocol deviations and it is therefore impossible to say whether an ITT analysis was performed. In view of these problems this study cannot be regarded as being of high methodological quality, although this may be revised if and when further information becomes available.

\section{Clinical Nurse Specialist intervention}

The Maughan 2001 study was very small and there is no record of any power calculation. Although the description of allocation was adequate, the method of randomisation does not appear to have attempted to stratify for the confounding effects of tumour sites and adjuvant therapies. As a result there was an imbalance between the control and intervention arms sufficient to cancel out any treatment effect. There was no description of the allocation concealment and the description of the study suggests that it was not used and should be graded D. The running of a parallel qualitative study was a potentially important source of bias since the qualitative assessment was also not balanced between the trial arms and it could potentially have influenced outcomes. As the assessment of the trial outcomes was by means of mailed back selfreport questionnaires, the blinding of outcome assessors was not required. Two patients who died (one in each arm) were excluded from the analysis of the trial and the analysis was therefore not by ITT. Re-analysis after substitution for the missing patients did not make any significant difference to the conclusions. Because of serious potential biases this study must be regarded as being of poor methodological quality.

\section{Psychoeducational group therapy}

This study does not provide detail of the randomisation process other than referring to a random number table. The concealment of allocation is unclear at best and was graded C. Although most of the assessments were by means of self-report questionnaires, it is unclear whether there was adequate blinding of outcome assessors for one of the outcomes, that of compliance with vaginal dilation. As the latter was not an outcome of interest for this study, this doubt does not therefore affect significantly the reliability of this study. The major methodological flaw with this study is the extremely high drop out rate (20\%, 3 controls, 5 interventions) that in the authors' own words means that this trial ight better be described as a non-equivalent controlled trial. Although some of these dropouts did return their initial baseline questionnaires these were not included in the analysis and therefore the analysis was not by intention to treat. The investigators carried out an analysis of baseline equivalence and found that the baseline SHF-GS scores were significantly lower in the experimental group (mean difference $=-0.132, \mathrm{t}(29)=3.41, \mathrm{p}=0.0019)$. This trial should be regarded as of very poor methodological quality and the results interpreted with great caution.

\section{Couple coping Intervention}

The main methodological difficulty with this study in terms of this review is that it has not been possible to distinguish the outcomes for gynaecological cancer patients from those for breast cancer patients. As a result all conclusions must refer to both groups and since gynaecological cancers were the minority it is possible that a strong effect in breast cancer patients could conceal no effect in gynaecological patients and vice versa. The method of randomisation is unclear and no method of concealment of allocation appears to have been undertaken (Grade D). Blinding of the assessment of outcomes was achieved by the use of self-report questionnaires. There was an extremely high dropout rate $(21 \%$ of women and $30 \%$ of their partners) and there is no information provided on whether this was evenly distributed across the study arms. The analysis was not by intention to treat and because of the limited data published it was not possible to re-analyse using substitution of the missing data. On the basis of the information available this trial was of very poor methodological quality.

\section{Effects of interventions}

The five studies in this review all trialed different interventions and did not utilise the same outcome measures and so cannot be directly compared. Meta-analysis is therefore not appropriate and the results of the studies will be presented individually.

\section{Topical oestrogen prophylaxis for post-irradiation vaginal complications}

A smaller proportion of those in the intervention group reported dyspareunia (6/44) than in the placebo group (16/49) but although the authors reported that this did not reach statistical significance with chi-square testing $(P=0.09)$, our analysis suggested that there was a significant difference with an OR of $0.33(95 \% \mathrm{Cl} 0.11$ to 0.93) (Analysis 1.1). Among the 26 patients in the intervention group who were sexually active, the majority (20) reported no dyspareunia and the remainder reported mild dyspareunia only. Of the 30 in the control arm reported to be sexually active, 14 denied dyspareunia, 16 reported dyspareunia, of whom 6 graded it as severe. When the sexually active participants were analysed separately with dyspareunia as an outcome measure, a greater treatment effect was demonstrated ( $\mathrm{OR}=0.26,95 \% \mathrm{Cl} 0.08$ to 0.84 ) (Analysis 1.2). As the follow-up in this study lasted only slightly longer than the treatment (mean 6.9 months) a temporary effect that was not maintained after treatment ended cannot be excluded. There were no losses to follow-up reported.

\section{Low dose-rate brachytherapy regimens in cervix cancer}

As the study of vaginal oestrogen above, this report has no information about pre-morbid sexual function and determines complications according to those reported to clinicians at followup visits. No losses to follow-up were reported but the paper does not explicitly state that there were none. The only outcome measure to relate to sexual function is that of dyspareunia and no data is included regarding the proportion who were sexually active. The overall prevalence of dyspareunia is low (11.8\%) but other gynaecological morbidities are reported such as pelvic sclerosis, incontinence, fistula and vaginal necrosis, all of which would significantly impair sexual function. Overall prevalence of dyspareunia was significantly lower in the $0.4 \mathrm{~Gy} / \mathrm{hr}$ group (OR = $0.37,95 \% \mathrm{Cl} 0.15$ to 0.93 ) Analysis 2.1. The paper also states that dyspareunia had resolved in the majority of both groups by 25 months after treatment and by this stage the differences were no longer significant $(\mathrm{OR}=0.39,95 \% \mathrm{Cl} 0.07$ to 2.05) Analysis 2.2. These findings are consistent with an amelioration of short-term sideeffects of brachytherapy without a significant long-term benefit.

\section{Clinical Nurse Specialist intervention}

Subjects were assessed at 6,12 and 24 weeks post surgery using the EORTC QLQ-30 and the Lasry Sexual Functioning Scale, both validated instruments. Unfortunately, no score data was 
published and our efforts to obtain this are ongoing. The authors report no significant differences between the groups in any of the sexual functioning scales examined and although the rates of resumption of intercourse in those previously sexually active showed a trend towards benefit this was not statistically significant ( $\mathrm{OR}=0.63,95 \% \mathrm{Cl} 0.17$ to 2.36 ) Analysis 3.1 . Significantly less of the intervention group (2/10 versus 9/10) had diminished satisfaction with intercourse six months after their surgery $(\mathrm{OR}=0.03,95 \% \mathrm{Cl}$ 0.00 to 0.37 ) Analysis 3.2 but it should be noted that there was an excess of patients having adjuvant therapy in the control group. These women might well have had a much shorter treatment-free interval and could well be expected to be at an earlier stage in their recovery than women who had surgery alone. Few conclusions should be drawn from this, especially given the very small numbers involved. The only losses to follow-up were because of death, suggesting that this intervention is acceptable to patients.

\section{Psychoeducational group therapy}

The mean pre-treatment SHF-GS for the intervention group was $0.401(S D=0.081)$ and that of the control $0.513(S D=0.126)$, both of which are comparable with the norms for healthy women. The SHFGS data for follow-up visits are only reported in graphical form with the comment that there was no evidence that the intervention had an impact on post intervention scores. This statement is prefaced with "After controlling for baseline scores..." so additional data is being sought from the authors to see how this was carried out. Although the trial did seem to demonstrate an effect on improved compliance with dilation in those followed up, the drop-out rate of $20 \%$ may indicate that either the therapy or the use of dilators was unacceptable to a significant minority. The effect on compliance did not translate into any improvement in sexual function.

\section{Couple-coping intervention}

This study excluded those who had a psychological disorder within the past year as well as those who were not in a committed relationship, raising doubts about whether any conclusions can be generalised. The authors did not present the data for gynaecological cancer patients separately and this additional information has not yet been received, so no analysis of this study has been possible. Overall participants in the trial showed no significant difference in all subscales bar the BISF Sexual Intimacy subscale where women who received CanCope reported less of a decrease in sexual intimacy (mean $=-1.21, \mathrm{SD}=2.5$ ) than women receiving medical information education (mean $=-3.53, \mathrm{SD}=2.55$ ) or patient coping training (mean $=-2.36, \mathrm{SD}=2.53$ ). As there appeared to be no significant effect on overall sexual function, the significance of this effect on intimacy is questionable.

\section{DISCUSSION}

This review has highlighted the difficulty in making evidence-based treatment decisions in this field. Five eligible trials were identified, most small, and all with poor methodology. Only two showed any demonstrable treatment effect but as one of these refers to a treatment modality that is not in widespread use, only one study has potential for widespread clinical application.

Vaginal oestrogen cream is a simple treatment which showed a short-term benefit in cervix cancer patients (Pitkin 1971). Unfortunately, as the numbers of cervix cancers are reduced by screening in developed countries, corpus cancer, whose incidence is rising, is becoming the most common indication for vaginal brachytherapy. Oestrogens are generally avoided in corpus cancer because of concerns that it may stimulate growth of the tumour cells which often have oestrogen receptors. Vaginal oestrogen is therefore likely to be of benefit to a only small subset of gynaecological cancer sufferers.

Low dose-rate brachytherapy prior to Radical Hysterectomy may be associated with a lower rate of dyspareunia in the short term (Haie-Meder 1994). Although a long-term benefit would be desirable even a short-term reduction of dyspareunia may help in reducing long-term psychosexual difficulties. This finding is of little utility to UK clinicians who do not commonly give preoperative radiotherapy for operable cervical cancers and since high dose-rates are generally used for brachytherapy performed as part of radical radiotherapy, these findings are not applicable in UK practice.

Clinical Nurse Specialists are an integral part of the Gynaecological Oncology Multidisciplinary Team in the UK. Their unique role puts them in an ideal position to be the first point of contact for gynaecological cancer patients regarding psychosexual difficulties. Maughan's study (Maughan 2001) is to be applauded for seeking to develop an evidence base for this work but trials many times the size of this one will be needed to give adequate power. This study does illustrate the difficulties that such studies will have in ensuring proper blinding.

A psychoeducational group therapy has demonstrated increased compliance with vaginal dilators but this did not translate into benefit in terms of a significant improvement in SHF-GS scores (Robinson 1999). This finding is unsurprising as dilation itself merely maintains anatomical normality and does not address the complex psychosocial issues that treatment for gynaecological cancer involves. Anatomical preservation is, however, an advantage in restoring sexual function so this intervention may be worthwhile as part of a package of measures addressing sexual function but this is outside the scope of this review.

Generally interventions that treat couples as opposed to individuals might be more successful in restoring a satisfactory sexual relationship. The absence of demonstrable benefit in this study of a couple-coping intervention (Scott 2004) should not discourage further research on couple-based interventions. Investigative research into the role of the partner of gynaecological cancer patients may provide directions for potential interventions.

It is disappointing how few RCTs were found in this review. This does raise the question of whether the ascertainment of trials was complete and whether there are more eligible trials that have simply failed to be found by this review. Further sources of studies include foreign language databases and abstracts of conference proceedings but there is often a law of diminishing returns with ever-wider searches. Given that the search so far has revealed so few eligible studies, it is doubtful but possible that further studies exist.

There is no shortage of interventions to be trialed as we identified a number of studies that, being non-RCTs, were ineligible for this review. Capone et al performed a non-RCT of 56 patients who received individual counselling during their hospital stay from a psychologist, incorporating a sexual rehabilitation component for 
those who had been sexually active (Capone 1980). Compared to a historical control group of 41 patients, a significantly higher proportion of those counselled had resumed their previous frequency of intercourse up to 12 months after treatment $(84 \%$ versus $42.9 \%, p<0.05$ ). The role of vaginal stent dilation for those who have had radiotherapy was explored in one study of 35 patients given a perspex stent after treatment (Decruze 1999). A lower incidence of vaginal stenosis as assessed by clinical examination was found in this group compared to a historical control group who had simply been advised to have regular intercourse. No outcomes in terms of sexual function were reported. The diversity of interventions identified in this review demonstrates that the management of sexual dysfunction requires attention to many factors, physical, psychological and social and future research should take this into account.

So why have so few RCTs been performed in this area? There is little doubt that this is an extremely sensitive area of which few have made a specialty in the past. While Clinical Psychologists and Psychosexual Therapists would undoubtedly have an interest in these problems, in some healthcare systems e.g. the UK, these services may not be sufficiently resourced as yet for most patients to be referred to them. Furthermore, these services are usually set up to deal with women and couples who do not have cancer and may not be in a position to offer specialised care for the problems that are specific to women who have been treated for a gynaecological cancer. In the UK, Clinical Nurse Specialists are now an essential part of the multidisciplinary teams treating gynaecological cancer and see psychosexual care as an integral part of their role. It is encouraging to note that one of the included studies was carried out by a Clinical Nurse Specialist and this may be the route by which future RCTs are organised.

As this review demonstrates, a good quality RCT is not an easy thing to carry out in this field and requires considerable support from clinical and non-clinical staff. There is an urgent need to develop suitable interventions that can be trialled since, as has been demonstrated by this review, there are very few tools available for the treatment of psychosexual morbidity in this group of women. Trials of psychological interventions are needed and as such interventions often require considerable amounts of the time of highly skilled practitioners, these trials are likely to be expensive and difficult to organise. It may be worthwhile for future trials to be organised on a multi-centre basis as this enables a number of centres to share resources and is more likely to attract the necessary funding.

The poor methodological quality of all the trials in this review is also disappointing. There is no doubt that the multi-factorial aetiology of psychosexual dysfunction means that there is a wealth of potential confounding factors to be considered by investigators of potential treatments. The consistent use of the same outcome measures in future trials would benefit future meta-analysis of results and this could perhaps be promoted by a group such as the International Gynaecological Cancer Society which could also provide the numbers required to achieve statistical power.

Since there may be significant differences between patients with different tumour sites, future trials should, where possible, be restricted to individual tumour sites. Where this is not possible, the trial should be powered for subgroup analysis by tumour site as an intervention that works well with a 40 year old cervical cancer patient may not work well with a 65 year old ovarian cancer patient.

Particular attention should also be given to ensuring adequate blinding in future studies to avoid potential bias. This may be particularly difficult with psychotherapies, since the duration and nature of the interaction with the therapist may reveal the allocation, but this bias could be avoided by having outcomes assessed by a third party observer, unaware of treatment allocation. With a number of the trials follow-up was up to 12 months and in two studies the benefit from treatment seemed to reduce with time. It is important that future studies ensure sufficiently long-term follow-up so that short-term effects can be identified.

It is clear from the results section of this review that further information regarding on a number of the studies would be useful in interpreting both their validity and reliability. It is hoped that some of these trial authors will be located and that the information will be obtained to update the review further.

\section{AUTHORS' CONCLUSIONS}

\section{Implications for practice}

There is insufficient evidence to support or refute the use of any interventions for psychosexual dysfunction after gynaecological cancer.

\section{Implications for research}

There is a need for multi-centre RCTs with outcome measures that have been validated in gynaecological cancer patients. When considering interventions to trial, we would suggest that investigators should focus on interventions that can be delivered by existing members of the multidisciplinary team treating women with gynaecological cancers. It is more likely that such measures, if found effective, will be affordable and capable of being integrated into standard care. An international consensus on outcome measures would greatly facilitate the comparison of interventions in the future.

\section{ACKNOWLEDGEMENTS}

We would like to acknowledge the help of Susan Prosser and the Library staff at Singleton Hospital in obtaining articles, and Professor Wu Taixiang from the Chinese Cochrane Centre for assistance with translations. 


\section{R E F E R E N C E S}

\section{References to studies included in this review}

Haie-Meder 1994 \{published data only (unpublished sought but not used)\}

Haie-Meder C, Kramar A, Lambin P, Lancar R, Scalliet P, Bouzy, et al. Analysis of complications in a prospective randomized trial comparin two brachytherapy low dose rates in cervical carcinoma. International Journal of Radiation Oncology, Biology, Physics 1994;29(5):953-60. [MEDLINE: 8083096]

Maughan 2001 \{published data only (unpublished sought but not used)\}

Maughan $\mathrm{K}$, Clarke $\mathrm{C}$. The effect of a clinical nurse specialist in gynaecological oncology on quality of life and sexuality. Journal of Clinical Nursing 2001;10:221-9. [MEDLINE: 11820343]

Pitkin 1971 \{published data only (unpublished sought but not used)\}

Pitkin RM, VanVoorhis LW. Postirradiation vaginitis. An evaluation of prophylaxis with topical estrogen. Radiology 1971;99:417-21. [MEDLINE: 5553582555]

Robinson 1999 \{published data only (unpublished sought but not used)\}

Robinson JW. Faris PD. Scott CB. Psychoeducational group increases vaginal dilation for younger women and reduces sexual fears for women of all ages with gynecological carcinoma treated with radiotherapy. International Journal of Radiation Oncology, Biology, Physics 1999;44(3):497-506. [MEDLINE: 10348277]

\section{Scott 2004 \{published data only (unpublished sought but not} used)\}

Scott JL. Halford WK. Ward BG. United we stand? The effects of a couple-coping intervention on adjustment to early stage breast or gynecological cancer. Journal of Consulting \& Clinical Psychology 2004;72(6):1122-35. [MEDLINE: 15612858]

\section{References to studies excluded from this review}

Bergmark 1999 \{published data only\}

Bergmark K, Avall-Lundqvist E, Dickman PW, Henningsohn L, Steineck $G$. Vaginal changes and sexuality in women with a history of cervical cancer. New England Journal of Medicine 1999;340(18):1383-9. [MEDLINE: 10228188]

Booth 1996 \{published and unpublished data\}

* Booth K, Maguire P. A controlled study of counselling intervention with patients suffering from cervical cancer. RCN Annual Research Meeting Abstracts. 1996.

\section{Capone 1980 \{published data only\}}

Capone MA, Good RS, Westie KS, Jacobson AF. Psychosocial rehabilitation of gynecologic oncology patients. Archives of Physical Medicine and Rehabilitation 1980;61(3):128-32. [MEDLINE: 7369850]
Cartwright-Alcarese 1995 \{published data only\}

Cartwright-Alcarese F. Addressing sexual dysfunction following radiation therapy for a gynecologic malignancy. Radiation Oncology Nursing Forum 1995;8:1227-32. [MEDLINE: 8532547]

Decruze 1999 \{published data only\}

Decruze SB, Guthrie D, Magnani R. Prevention of vaginal stenosis in patients following vaginal brachytherapy. Clinical Oncology 1999;11:46-8. [MEDLINE: 10194586]

Gothard 2005 \{published data only\}

Gothard L, Cornes P, Brooker S, Earl J, Glees J, Hall E, et al. Phase II study of vitamin $E$ and pentoxifylline in patients with late side effects of pelvic radiotherapy. Radiotherapy \& Oncology 2005;75(3):334-41. [MEDLINE: 16086914]

Kikku 1982 \{published data only\}

Kilkku P, Gronroos M, Punnonen R. Sexual Function after Conization of the Uterine Cervix. Gynecologic Oncology 1982;14:209-12. [MEDLINE: 7129217]

\section{Landoni 1985 \{published data only\}}

Landoni F, Proserpio M, Maneo A, Cormio G, Zanetta G, Milani R. Repair of the perineal defect after radical vulvar surgery direct closure versus skin flaps reconstruction. A retrospective comparative study. Australian \& New Zealand Journal of Obstetrics \& Gynaecology 1995;35(3):300-4. [MEDLINE: 8546649]

Schroder 2005 \{published data only\} Schroder M, Mell LK, Hurteau JA, Collins YC, Rotmensch J, Waggoner SE, et al. Clitoral therapy device for treatment of sexual dysfunction in irradiated cervical cancer patients. International Journal of Radiation Oncology, Biology, Physics 2005;61(4):1078-86. [MEDLINE: 15752887]

\section{Additional references}

Anderson 1986

Anderson BL, Wolfe RM. Chronic physcial illness and sexual behaviour: psychological issues. Journal of Consulting and Clinical Psychology 1986;54:168-75.

\section{APA 1994}

American Psychiatric Association. Diagnostic and Statistical Manual of Mental Disorders DSM-IV-TR (Text Revision). American Psychiatric Press Inc, 1994.

\section{Bergmark 1999a}

Bergamrk K, Avall-lundqvist E, Dickman P, Henningsohn L, Steineck $G$. Vaginal changes and sexuality in women with a history of cervical cancer. The New England Journal of Medicine 1999;340(18):1383-9.

\section{Butler 1998}

Butler L, Banfield V, Sveinson T, Allen K. Issues of sexual health in cancer care. Western Journal of Nursing Research 1998;20(6):684-700. 


\section{Cochrane Handbook}

Higgins JPT, Green S (editors). Cochrane Handbook for Systematic Reviews of Interventions Version 5.0.0 [updated February 2008]. The Cochrane Collaboration, 2008. Available form www.cochrane-handbook.org.

\section{Corney 1992}

Corney RH, Everett H, Howells A, Crowther ME. Psychosocial adjustment following major gynaecological surgery for carcinoma of the cervix and vulva. Journal of psychosomatic research 1992;36(6):561-8.

\section{Corney 1993}

Corney RH, Crowther ME, Everett H, Howells A, Shepherd JH. Psychosexual dysfunction in women with gynaecological cancer following radical pelvic surgery. British Journal of Obstetrics and Gynaecology 1993;100(1):73-8.

\section{Crowther 1994}

Crowther ME, Corney RH, Shepherd JH. Psychosexual implications of gynaecological cancer. $B M J$ 1994;308(6933):869-70.

\section{Egger 1997}

Egger M, Davey Smith G, Schneider M, Minder C. Bias in meta-analysis detected by a simple, graphical test. BMJ 1997;315:629-34.

\section{Goldberg 1997}

Goldberg, D.P. The validity of two versions of the GHQ in the WHO study of mental illness in general health care. Psychological Medicine 1997;27:191-7.

\section{CHARACTERISTICS OF STUDIES}

Characteristics of included studies [ordered by study ID]

\section{Stead 1999}

Stead ML, Crocombe WD, Fallowfield LJ, Selby P, Perren TJ, Garry R, et al. Sexual activity questionnaires in clinical trials: acceptability to patients with gynaecological disorders. British Journal of Obstetrics and Gynaecology 1999;106(1):50-4.

\section{Steer 1999}

Steer RA, Cavalieri TA, Leonard DM. Use of the Beck Depression Inventory for Primary Care to screen for major depression disorders. General Hospital Psychiatry 1999;21:106-111.

\section{Thranov 1994}

Thranov I, Klee M. Sexuality among gynecologic cancer patients - a cross sectional study. Gynecologic Oncology 1994;52:14-9.

\section{Ware 1993}

Ware JE Jnr. SF-36 Health Survey manual and interpretation guide. Boston: The Health Institute, New England Medical Centre, 1993.

\section{Weijmar-Schultz 1990}

Weijmar-Schultz WCM, van de Wiel HBM, Bouma J, Janssens J, Littlewood J. Psychosexual functioning after the treatment of cancer of the vulva. Cancer 1990;66:402-7.

\section{Zigmond 1983}

Zigmond AS, Snaith RP. The Hospital Anxiety and Depression Scale. Acta Psychiatrica Scandinavica 1983;67:361-70.

* Indicates the major publication for the study

Haie-Meder 1994

\begin{tabular}{ll}
\hline Methods & RCT with local control rate, overall and progression-free survival as primary endpoints \\
\hline Participants & Pre-operative patients with Stage I-IIA cervix cancer (n=204) \\
\hline Interventions & Neoadjuvant low dose-rate brachytherapy at 0.4Gy/hr versus 0.8Gy/hr \\
\hline Outcomes & Dyspareunia recorded in a physician-administered questionnaire \\
\hline Notes & Modality not used in some countries including UK. Randomisation method not described. \\
\hline Risk of bias & Authors' judgement Support for judgement \\
\hline $\begin{array}{l}\text { Bias } \\
\text { Allocation concealment } \\
\text { (selection bias) }\end{array}$ & Low risk Adequate \\
\hline $\begin{array}{l}\text { Blinding (performance } \\
\text { bias and detection bias) } \\
\text { All outcomes }\end{array}$ & Low risk \\
\hline
\end{tabular}


Haie-Meder 1994 (Continued)

Other bias High risk Some relevant baseline characteristics not reported.

Maughan 2001

\begin{tabular}{ll}
\hline Methods & RCT performed alongside qualitative study \\
\hline Participants & Women undergoing treatment for a variety of gynaecological cancers $(\mathrm{n}=36)$ \\
\hline Interventions & One pre-surgery consultation and 3 home visits by Nurse Specialist versus standard nursing care \\
\hline Outcomes & Self-report questionnaires (EORTC QLQ-30, Lasry Sexual Functioning Scale) \\
\hline Notes & $\begin{array}{l}\text { Significant differences between groups in terms of cancer treatment received. Adequate randomisation } \\
\text { method. }\end{array}$
\end{tabular}

\section{Risk of bias}

\begin{tabular}{|c|c|c|}
\hline Bias & Authors' judgement & Support for judgement \\
\hline $\begin{array}{l}\text { Random sequence genera- } \\
\text { tion (selection bias) }\end{array}$ & Low risk & \\
\hline $\begin{array}{l}\text { Allocation concealment } \\
\text { (selection bias) }\end{array}$ & Low risk & \\
\hline $\begin{array}{l}\text { Blinding (performance } \\
\text { bias and detection bias) } \\
\text { All outcomes }\end{array}$ & High risk & \\
\hline $\begin{array}{l}\text { Incomplete outcome data } \\
\text { (attrition bias) } \\
\text { All outcomes }\end{array}$ & Low risk & \\
\hline Other bias & High risk & Not intention-to-treat analysis. \\
\hline
\end{tabular}

\section{Pitkin 1971}

\begin{tabular}{ll}
\hline Methods & Placebo-controlled trial \\
\hline Participants & Women with no residual disease after radiotherapy for cervix cancer stages I-IV (n=93) \\
\hline Interventions & Vaginal oestrogen cream versus placebo cream \\
\hline Outcomes & Self-report of dyspareunia \\
\hline Notes & Short follow-up (range 5-8 months, median 6.9). Inadequate description of randomisation. \\
\hline Risk of bias & Authors' judgement Support for judgement \\
\hline Bias &
\end{tabular}


Pitkin 1971 (Continued)

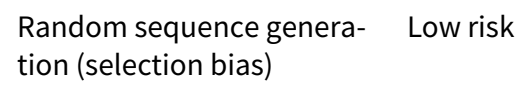

\begin{tabular}{lll}
\hline $\begin{array}{l}\text { Allocation concealment } \\
\text { (selection bias) }\end{array}$ & Low risk - adequate
\end{tabular}

\begin{tabular}{|c|c|}
\hline $\begin{array}{l}\text { Blinding (performance } \\
\text { bias and detection bias) } \\
\text { All outcomes }\end{array}$ & Low risk \\
\hline $\begin{array}{l}\text { Incomplete outcome data } \\
\text { (attrition bias) } \\
\text { All outcomes }\end{array}$ & Low risk \\
\hline $\begin{array}{l}\text { Selective reporting (re- } \\
\text { porting bias) }\end{array}$ & Low risk \\
\hline Other bias & Low risk \\
\hline
\end{tabular}

\section{Robinson 1999}

\begin{tabular}{ll}
\hline Methods & Randomised controlled trial \\
\hline Participants & Women having pelvic radiotherapy for cervix or endometrial cancers, stages I-II ( $\mathrm{n}=40)$ \\
\hline Interventions & Two 1.5 hour counselling sessions versus single meeting with counsellor + leaflet. \\
\hline Outcomes & Sexual History Form Global Score (SHF-GS) and self-report compliance with vaginal dilation \\
\hline Notes & Only 32 patients analysed - not intention to treat. Partial description of randomisation process.
\end{tabular}

\section{Risk of bias}

\begin{tabular}{lll}
\hline Bias & Authors' judgement & Support for judgement \\
\hline $\begin{array}{l}\text { Random sequence genera- } \\
\text { tion (selection bias) }\end{array}$ & Low risk & \\
\hline $\begin{array}{l}\text { Allocation concealment } \\
\text { (selection bias) }\end{array}$ & Unclear risk & C-Inadequate \\
\hline $\begin{array}{l}\text { Incomplete outcome data } \\
\text { (attrition bias) }\end{array}$ & High risk \\
All outcomes & \\
\hline $\begin{array}{l}\text { Selective reporting (re- } \\
\text { porting bias) }\end{array}$ & High risk \\
\hline
\end{tabular}

Methods Randomised controlled trial


Scott 2004 (Continued)
Participants
37 women undergoing treatment for gynaecological cancer and their partners

Interventions

Couple-Coping Therapy ( $5 \times 2 \mathrm{hr}$ sessions at home and $2 \times 30 \mathrm{~min}$ phone calls) versus Medical Information Education (Booklet; $5 \times 15 \mathrm{~min}$ phone calls) versus Patient Coping Training (4x2hr sessions at home; 2x30min phone calls)

\begin{tabular}{ll}
\hline Outcomes & Brief Index of Sexual Functioning (BISF) self-report scale \\
\hline Notes & Randomisation method not described. High proportion lost to follow-up.
\end{tabular}

\section{Risk of bias}

\begin{tabular}{lll}
\hline Bias & Authors' judgement & Support for judgement \\
\hline $\begin{array}{l}\text { Allocation concealment } \\
\text { (selection bias) }\end{array}$ & High risk & C - Inadequate \\
\hline $\begin{array}{l}\text { Blinding (performance } \\
\text { bias and detection bias) } \\
\text { All outcomes }\end{array}$ & High risk \\
\hline $\begin{array}{l}\text { Incomplete outcome data } \\
\text { (attrition bias) }\end{array}$ & High risk \\
$\begin{array}{l}\text { All outcomes } \\
\text { Selective reporting (re- } \\
\text { porting bias) }\end{array}$ & High risk \\
\hline
\end{tabular}

Characteristics of excluded studies [ordered by study ID]

\begin{tabular}{ll}
\hline Study & Reason for exclusion \\
\hline Bergmark 1999 & Retrospective descriptive study \\
\hline Booth 1996 & No outcome measure relating to sexual function \\
\hline Capone 1980 & Non-randomised study using historical controls \\
\hline Cartwright-Alcarese 1995 & Non-systematic review \\
\hline Decruze 1999 & Non-randomised study using historical controls \\
\hline Gothard 2005 & Non-randomised Phase Il study \\
\hline Kikku 1982 & Uncontrolled study \\
\hline Landoni 1985 & Non-randomised study using historical controls \\
\hline Schroder 2005 & Uncontrolled Phase II study \\
\hline
\end{tabular}


DATA AND ANALYSES

Comparison 1. Vaginal oestrogen versus placebo

\begin{tabular}{lllll}
\hline Outcome or subgroup title & No. of studies & $\begin{array}{l}\text { No. of partici- } \\
\text { pants }\end{array}$ & Statistical method & Effect size \\
\hline 1 Dyspareunia in all patients & 1 & 93 & $\begin{array}{l}\text { Odds Ratio (M-H, Fixed, 95\% } \\
\text { Cl) }\end{array}$ & $0.33[0.11,0.93]$ \\
\hline 2 Dyspareunia in sexually active & 1 & 56 & $\begin{array}{l}\text { Odds Ratio (M-H, Fixed, 95\% } \\
\text { Cl) }\end{array}$ & $0.26[0.08,0.84]$ \\
\hline
\end{tabular}

Analysis 1.1. Comparison 1 Vaginal oestrogen versus placebo, Outcome 1 Dyspareunia in all patients.

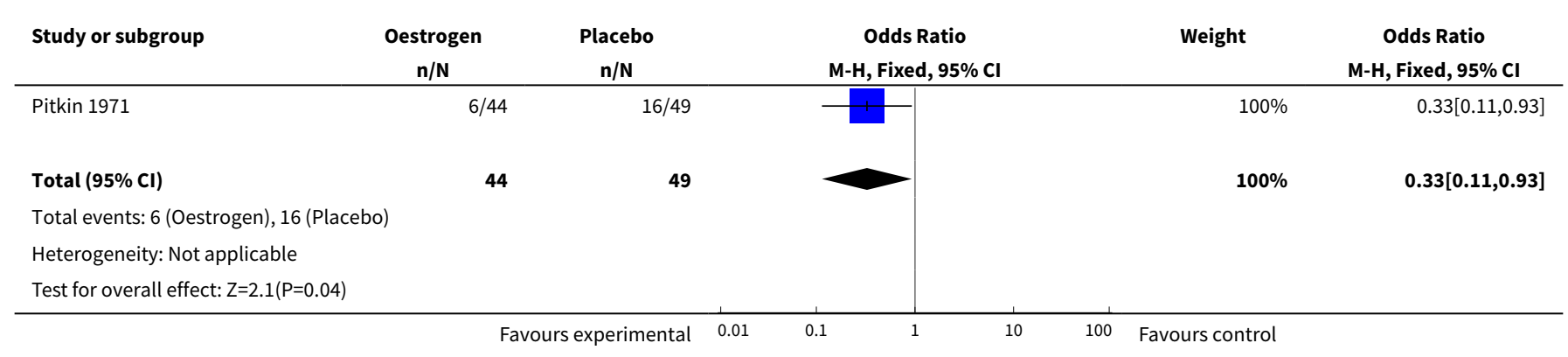

Analysis 1.2. Comparison 1 Vaginal oestrogen versus placebo, Outcome 2 Dyspareunia in sexually active.

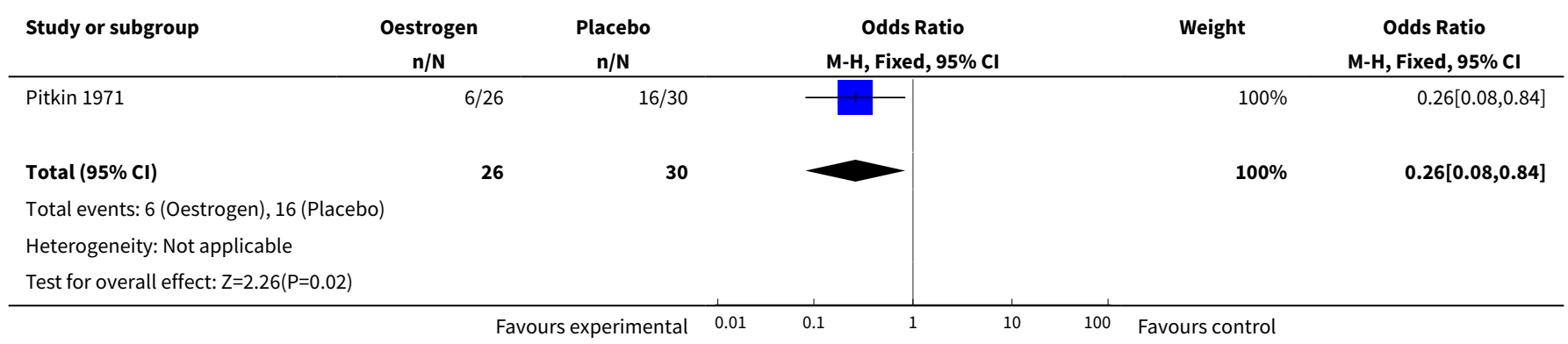

\section{Comparison 2. Brachytherapy $0.4 \mathrm{~Gy} / \mathrm{Hr}$ versus $0.8 \mathrm{~Gy} / \mathrm{hr}$}

\begin{tabular}{|c|c|c|c|c|}
\hline Outcome or subgroup title & No. of studies & $\begin{array}{l}\text { No. of partici- } \\
\text { pants }\end{array}$ & Statistical method & Effect size \\
\hline 1 Dyspareunia in all patients & 1 & 204 & $\begin{array}{l}\text { Odds Ratio (M-H, Fixed, 95\% } \\
\mathrm{Cl} \text { ) }\end{array}$ & $0.37[0.15,0.93]$ \\
\hline $\begin{array}{l}2 \text { Dyspareunia at } 25 \text { months post } \\
\text { treatment }\end{array}$ & 1 & 204 & $\begin{array}{l}\text { Odds Ratio (M-H, Fixed, 95\% } \\
\mathrm{Cl})\end{array}$ & $0.39[0.07,2.05]$ \\
\hline
\end{tabular}


Analysis 2.1. Comparison 2 Brachytherapy $0.4 \mathrm{~Gy} / \mathrm{Hr}$ versus $0.8 \mathrm{~Gy} / \mathrm{hr}$, Outcome 1 Dyspareunia in all patients.

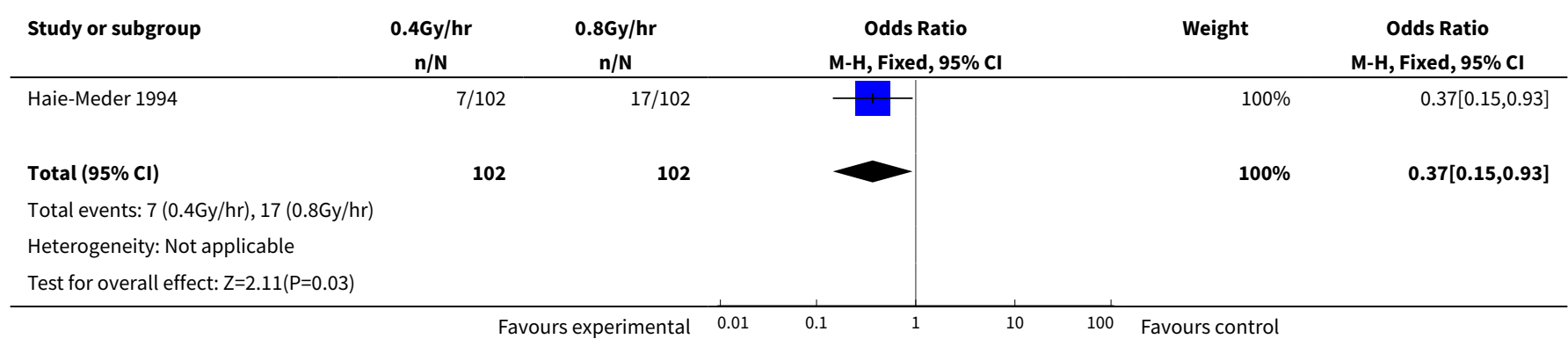

Analysis 2.2. Comparison 2 Brachytherapy $0.4 \mathrm{~Gy} / \mathrm{Hr}$ versus

$0.8 \mathrm{~Gy} / \mathrm{hr}$, Outcome 2 Dyspareunia at 25 months post treatment.

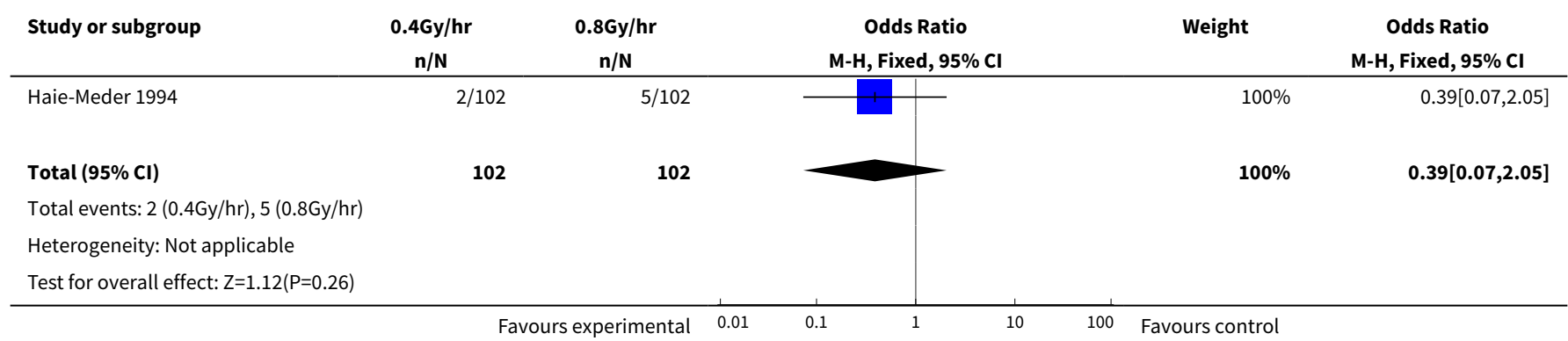

Comparison 3. Clinical Nurse Specialist versus Standard Care

\begin{tabular}{|c|c|c|c|c|}
\hline Outcome or subgroup title & No. of studies & $\begin{array}{l}\text { No. of partici- } \\
\text { pants }\end{array}$ & Statistical method & Effect size \\
\hline 1 Not sexually active & 1 & 36 & $\begin{array}{l}\text { Odds Ratio (M-H, Fixed, 95\% } \\
\mathrm{Cl} \text { ) }\end{array}$ & $0.63[0.17,2.36]$ \\
\hline $\begin{array}{l}2 \text { Previously active, unsatisfactory } \\
\text { now }\end{array}$ & 1 & 20 & $\begin{array}{l}\text { Odds Ratio (M-H, Fixed, 95\% } \\
\mathrm{Cl} \text { ) }\end{array}$ & $0.03[0.00,0.37]$ \\
\hline
\end{tabular}

Analysis 3.1. Comparison 3 Clinical Nurse Specialist versus Standard Care, Outcome 1 Not sexually active.

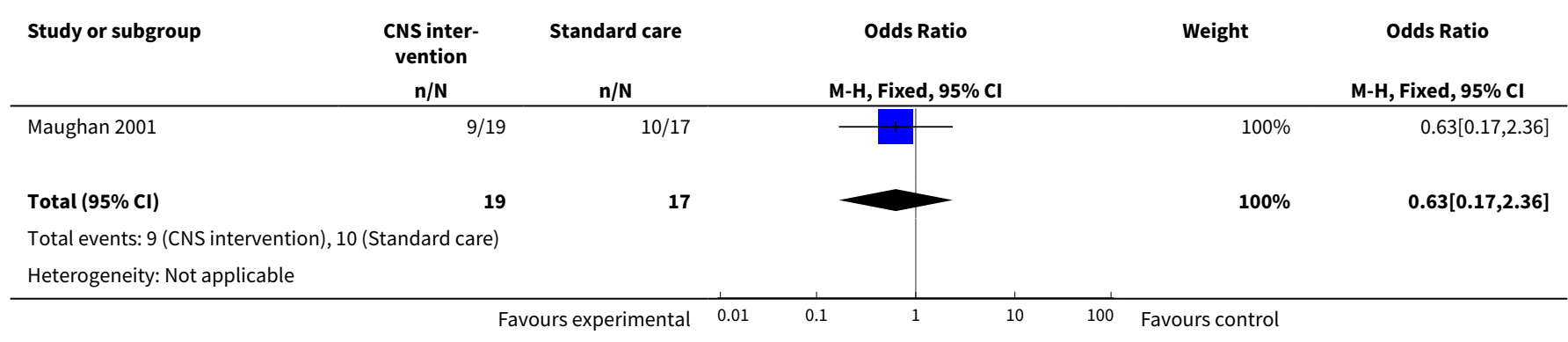




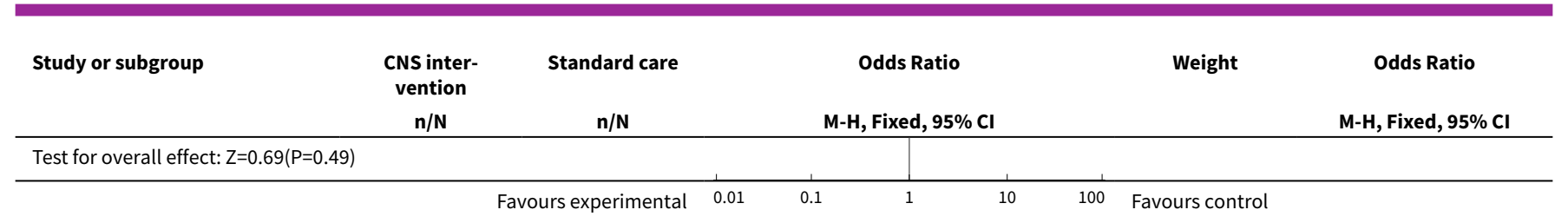

Analysis 3.2. Comparison 3 Clinical Nurse Specialist versus Standard Care, Outcome 2 Previously active, unsatisfactory now.

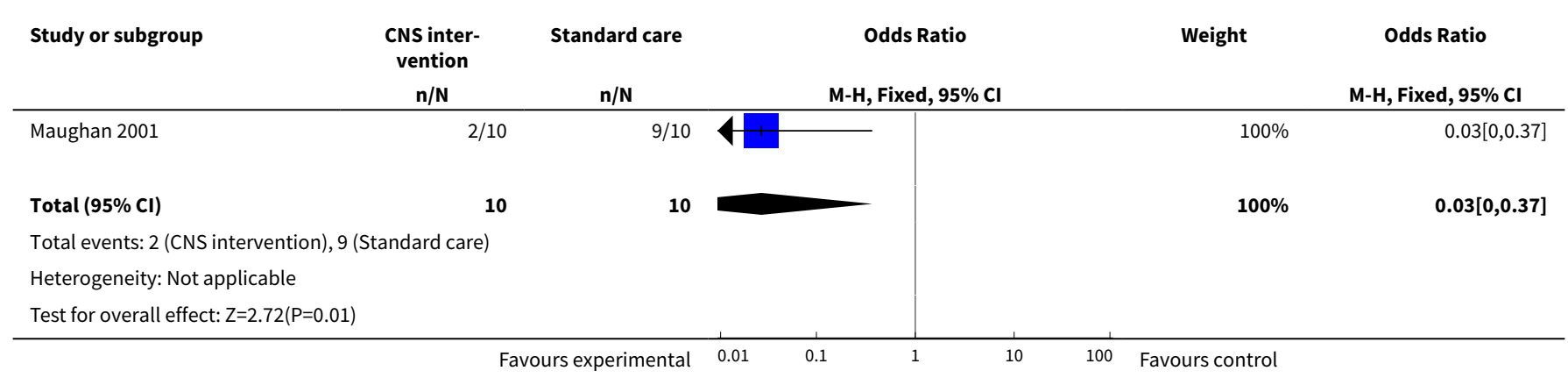

\section{APPENDICES}

\section{Appendix 1. Search Strategy for OvidSP}

1. Randomized controlled trial.pt.

2. controlled clinical trial.pt.

3. randomized.ab.

4. placebo.ab.

5. drug therapy.fs.

6. randomly.ab.

7. trial.ab.

8. groups.ab.

9. 1 or 2 or 3 or 4 or 5 or 6 or 7 or 8

10 humans.sh.

11. 9 and 10

12. Gyn?ecologi\$.mp.

13. Female genital.mp.

14. Ovar\$.mp.

15. Fallopian tube.mp.

16. Uter\$.mp.

17. Corpus.mp.

18. Endometri\$.mp.

19. Cervi\$.mp.

20. Vagin\$.mp.

21. Vulv\$.mp.

22. Cancer.mp.

23. Carcinoma.mp.

24. Sarcoma.mp.

25. Malignancy.mp.

26. exp Genital Neoplasms, Female/

27. exp Sexual Dysfunctions, Psychological/

28. Dyspareunia/

29. Sexual.mp.

30. Psychosexual.mp. 
31. Intercourse.mp.

32. Dyspar?unia.mp.

33. Orgas\$.mp.

34. cognitive behavio? ral therapy.mp.

35. (cognitive adj2 therapy).mp.

36. counsel?ing.mp.

37. psychodynamic.mp.

38. relaxation therapy.mp.

39. desensiti?ation.mp.

40. rational emotive therapy.mp.

41. antidepressant?.mp.

42. sildenafil.mp.

43. 12 or 13 or 14 or 15 or 16 or 17 or 18 or 19 or 20 or 21

44. 22 or 23 or 24 or 25

45. 43 and 44

46. 45 or 26

47. 27 or 28 or 29 or 30 or 31 or 32 or 33

48. 46 and 47 and 11

Key - $\mathrm{mp}=$ title, original title, abstract, name of substance word, subject heading word

\section{WHAT'S NEW}

\begin{tabular}{lll}
\hline Date & Event & Description \\
\hline 5 September 2019 & Amended & $\begin{array}{l}\text { This Cochrane Review will be superseded by a new protocol and } \\
\text { review currently under development, which will include updated } \\
\text { Cochrane methods. }\end{array}$ \\
\hline
\end{tabular}

\section{H I S T O RY}

Protocol first published: Issue 2, 2004

Review first published: Issue 2, 2009

\begin{tabular}{lll}
\hline Date & Event & Description \\
\hline 3 September 2018 & Amended & $\begin{array}{l}\text { New contributors required to update and maintain this Cochrane } \\
\text { Review. }\end{array}$ \\
\hline 27 April 2009 & Amended & SS statement added \\
\hline 28 January 2009 & Amended & Peer review comments incorporated. \\
\hline 6 November 2008 & Amended & Converted to new review format. \\
\hline
\end{tabular}

\section{CONTRIBUTIONS OF AUTHORS}

FK and PF developed the outline protocol, carried out the searches and assessments and wrote the review. SK edited the protocol and the review.

\section{DECLARATIONS OF INTEREST}

Nil known 


\section{DIFFERENCES BETWEEN PROTOCOL AND REVIEW}

The protocol planned that conference abstracts would be hand searched for relevant studies. This was deemed unnecessary as the abstracts for the relevant societies are published in their associated journals and were thus accessible to the electronic searches.

\section{IN DEX TERMS}

\section{Medical Subject Headings (MeSH)}

Brachytherapy; Estrogens [therapeutic use]; Genital Neoplasms, Female [psychology] [therapy]; Patient Education as Topic; Psychotherapy [methods]; Randomized Controlled Trials as Topic; Sexual Dysfunctions, Psychological [etiology] [therapy]

\section{MeSH check words}

Female; Humans 